\title{
Identification of Human Joint Mechanical Properties from Single Trial Data
}

\author{
Yangming Xu, Member, IEEE, and John M. Hollerbach,* Fellow, IEEE
}

\begin{abstract}
A method is presented for estimating the timevarying compliance parameters of the elbow joint from a single movement. The method separates by frequency the perturbed from the voluntary response, then determines the parameters by exponentially weighted least squares. The tracking performance of the method is established by simulation and by a calibrated mechanical joint. Experimental results are presented on timevarying posture and slow movement.
\end{abstract}

Index Terms-Arm movements, frequency separation, human joint dynamics, perturbation analysis, system identification.

\section{INTRODUCTION}

$\mathbf{I}$ DENTIFYING the mechanical properties of the human neuromusculoskeleton system under normal conditions is challenging because of the time-varying nature of the system, nonlinearities, unsensed voluntary inputs, and adaptation of the central nervous system. Past approaches have relied on apparatuses using electrical or hydraulic actuators that constrain natural human joint movements and limit the results under these conditions. However, the constraint also allows time-invariant methods to be used because the human joint is forced to operate under some fixed operating condition. The time-invariant methods include frequency methods (transfer function), pulse response methods, and a sinusoidals method [7]-[9], [11]. The time-invariant results obtained are valid only for a fixed operating point, and the transient properties of the human joint cannot be obtained with these methods directly.

More recently, a static nonlinear, time-invariant method has been applied to identify the different contributions to the total joint mechanical properties of the reflex versus intrinsic components [15] by using random position perturbations with a hydraulic apparatus. Again, the approach takes advantage of the constraints of the apparatus on the human joint to obtain tonic contractions.

To overcome the motion limitations of past apparatuses, we have developed nonrestrictive one-dimensional (1-D) and twodimensional (2-D) airjet systems capable of applying random force perturbations to the human joints without any mechanical constraints [18], [21]. The perturbations can be continuously applied to the human joints without impairing the natural movement. This significant advance in apparatuses brings

Manuscript received December 22, 1993; revised March 3, 1998. This work was supported by the Medical Research Council of Canada (MRC) and by NSERC NCE IRIS II Project AMD-2. Asterisk indicates corresponding author.

Y. Xu is with the Sarcos Research Corporation, Salt Lake City, UT 84108 USA.

*J. M. Hollerbach is with the Department of Computer Science, University of Utah, 50 S. Central Campus Drive, Room 3190, Salt Lake City, UT 84112 9205 USA (e-mail: jmh@cs.utah.edu).

Publisher Item Identifier S 0018-9294(98)05521-9. the possibility of identifying the time-varying human joint mechanical properties under natural movement.

One of the central issues in studying the mechanical properties of the human arm joints during movement using noninvasive methods is to separate the voluntary inputs and movements from the perturbation inputs and perturbed joint movements. Even though electromyograms are related to the voluntary actions, they are too noisy to provide reliable information of the voluntary movement. In essence, the voluntary movement is not measurable.

Ensemble methods are promising [3], [14] because the results at any instant of time are supported by more data points. The methods align many trials with similar movement profile based on a feature in the movement profile to calculate an average movement. The perturbed movement is obtained by subtracting the average movement from the movement measurement. Theoretically, they can track an instantaneous change of the system dynamics. One problem is intertrial variation; since the perturbation amplitude is small, the error due to the intertrial variation is often on the same order as the perturbation. Furthermore, the intertrial variation may neither be white nor Gaussian, and may even have nonzero mean. In order to have a stereotyped motion, most of the movements have to be fast. These methods fail for time-varying posture identification because there exists no features at all across different trials, therefore, the alignment cannot be done.

In this paper, we propose a method for slow time-varying posture and slow motion based on a single trial that is capable of identifying the time-varying joint mechanical properties and does not have the intertrial variation issue. The method separates the perturbed and voluntary responses in frequency, and then applies exponentially weighted least squares. It is called the frequency separation and exponentially weighted least squares (FSEWLS) method. The method requires only a single-movement trial data. In the following sections, we will first describe the theory for different measurement conditions and propose the frequency separation, then analyze the error and limitations, and finally demonstrate the results by applying it to data from simulation and experiments.

\section{THEORY}

\section{A. Linearized Human Forearm Model}

The equation for the human elbow joint under perturbation can be written as

$$
I \ddot{\theta}=\tau_{m}(\dot{\theta}, \theta, u)+\tau_{p}+\tau_{g}(\theta)
$$


where $I$ is the moment of inertia of the forearm, $\theta$ is the actual joint angle, $\tau_{m}(\dot{\theta}, \theta, u)$ is the total torque from the muscles and passive tissue, $u$ is the muscle input, $\tau_{p}$ is the perturbation torque, and $\tau_{g}(\theta)$ is the gravity induced torque. $\tau_{m}$ and $\tau_{g}$ are nonlinear and time varying. Because $\tau_{m}$ is not known, we linearize it around an unperturbed operating point $\left(\theta_{n}, u_{n}\right)$

$$
I(t) \delta \ddot{\theta}(t)+B(t) \delta \dot{\theta}(t)+K(t) \delta \theta(t)=\tau_{p}(t)
$$

where $\delta \theta=\theta-\theta_{n}, \delta u=u-u_{n}, B(t)=-\partial \tau_{m} / \partial \dot{\theta}$, and $K(t)=-\partial\left(\tau_{m}-\tau_{g}\right) / \partial \theta$. We have assumed a random external perturbation that is not tracked by voluntary effort. The compliance parameters $I(t), B(t), K(t)$ reflect the linearized joint mechanical properties at an unperturbed operating point; the validity of this linearization is tested in experiments. We measure $\theta_{\text {meas }}(t)=\theta_{n}(t)+\delta \theta(t)$ and $\tau_{\text {meas }}(t)=\tau_{n}(t)+$ $\tau_{p}(t)$, where $\tau_{n}$ is the force sensor signal induced by the voluntary movement.

\section{B. Removing Voluntary Movement}

An effective way to remove $\theta_{n}$ and $\tau_{n}$ from the measurements is to make the voluntary movement and the perturbation separable in frequency. The movement tasks considered in this paper are below $1 \mathrm{~Hz}$, while the perturbation has enough power at high frequencies. Using a zero-phase-shift acausal linear high-pass filter $H(s)$, we can extract the perturbation force and the perturbed response from the measurements

$$
\begin{aligned}
\delta \tilde{\theta}(t) & =H(s) \theta_{\text {meas }}(t)=H(s)\left[\theta_{n}(t)+\delta \theta(t)\right] \approx H(s) \delta \theta(t) \\
\tilde{\tau}(t) & =H(s) \tau_{\text {meas }}(t)=H(s)\left[\tau_{n}(t)+\tau(t)\right] \approx H(s) \tau(t) .
\end{aligned}
$$

Appendix B gives a qualitative error analysis due to high-pass filtering in respect to (2). A quantitative analysis is given in the simulation and calibration experiment later.

\section{Estimating Derivatives}

The derivatives of the position are not directly measured in the experiment. The differentiation of position data to estimate its derivatives is sensitive to noise in the position measurements and numerical error. One solution to this problem is to use a linear low-pass filter $L(s)$ to process the input and output measurements assuming the filter bandwidth is much higher than the variation of the parameters

$$
\begin{aligned}
& \delta \tilde{\theta}_{L}(t)=L(s) \delta \tilde{\theta}(t), \quad \delta \dot{\tilde{\theta}}_{L}(t)=s L(s) \delta \tilde{\theta}(t) \\
& \delta \ddot{\tilde{\theta}}_{L}(t)=s^{2} L(s) \delta \tilde{\theta}(t), \quad \tilde{\tau}_{L}(t)=L(s) \tilde{\tau}(t) .
\end{aligned}
$$

We let $L(s)=s^{n-2} a^{n} /(s+a)^{n}$, where the filter constant $a$ determines the filter response time and $n$ is the order of the filter. Appendix A shows the error due to low-pass filtering.

\section{Extracting Parameters}

We cast (2) into the regression form

$$
\hat{y}(t)=\Phi^{T}(t) \hat{\Theta}(t)
$$

where $\hat{y}(t)=\tilde{\tau}_{L}(T), \Phi(t)=\left\lfloor\delta \ddot{\tilde{\theta}}_{L}(t) \delta \dot{\tilde{\theta}}_{L}(t) \delta \tilde{\theta}_{L}(t)\right\rfloor$ and $\hat{\Theta}(t)=\left[\begin{array}{lll}\hat{I}(t) & \hat{B}(t) & \hat{K}(t)\end{array}\right\rfloor^{T}$. We extract $\Theta(t)$ using the

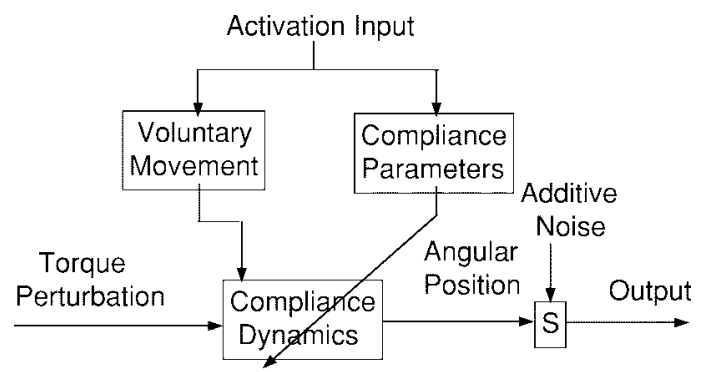

Fig. 1. Block diagram of the simulation procedure for identification of the time-varying elbow joint dynamics in posture and movement.

exponentially weighted least squares method [6], [12], [13]. The continuous domain equations are

$$
\begin{aligned}
& \dot{\hat{\Theta}}(t)=P(t) \Phi(t)\left(y(t)-\Phi^{T}(t) \hat{\Theta}(t)\right) \\
& \dot{P}(t)=-P(t) \Phi(t) \Phi^{T}(t) P(t)+\lambda P(t)
\end{aligned}
$$

where $\lambda$ is a weighting factor. The estimation error is related to the parameter variation rate and measurement noise [16].

In a discrete time domain implementation of (6) as in [1], [12], and [13], we found ill conditioning in transforming from discrete to continuous parameters because of the small sampling period. Consequently, we implement (6) in the continuous time domain, by using a fourth-order Runge-Kutta integration method with the step size equal to the sampling period. The points between the steps required by the Runge-Kutta method are obtained through linear interpolation. The continuous model gives accurate results especially at small sampling periods. The initial values for parameter estimates $\hat{\Theta}(0)$ and gain matrix $P(0)$ in (6) are calculated using standard least squares.

\section{Simulation StUdy}

\section{A. Simulation Method}

We first tested the method described in Section II with data generated by simulating the changes in the human elbow joint dynamics from our previous studies [3], [20], which include the stiffness and damping changes in posture and movement. Fig. 1 shows a block diagram of the simulation procedure.

The linearized compliance dynamics are similar to (2)

$$
I \ddot{\theta}+B \dot{\theta}+K \theta=\tau_{v}+\tau_{p}
$$

where $\tau_{v}$ is the joint torque due to the voluntary action, $\theta=\left(\theta_{n}+\delta \theta\right)$. On the basis of previous quasitime-invariant studies [3], [4], [11], [20], [22], the voluntary movement and stiffness $K$ are assumed to vary sinusoidally with the same frequency and with different amplitudes of $\pm 0.5 \mathrm{rad}$ and \pm 15 $\mathrm{Nm} / \mathrm{rad}$ plus a mean of $40 \mathrm{Nm} / \mathrm{rad}$, the inertia $I$ is assumed to be time-invariant, $0.06 \mathrm{Nms}^{-2} / \mathrm{rad}$, and the damping is assumed to vary similar to the stiffness while the damping ratio is assumed to be constant [9].

The force perturbation input is a pseudorandom binary force sequence (PRBS) with an amplitude of about $4 \mathrm{Nm}$, the same as the one used in the experiments. The autocorrelation is small (below 10\%) beyond 10-ms lag (ten sampling periods), which 
indicates that it is essentially an uncorrelated random process in the $100-\mathrm{Hz}$ frequency bandwidth. The power spectrum of the PRBS has useful power up to $50 \mathrm{~Hz}$. The input and output data are filtered by a low-pass filter with a cutoff frequency of $150 \mathrm{~Hz}$, which mimics the anti-aliasing filter used in the experiment.

We evaluate the accuracy of the estimated parameters by calculating the percentage of variance accounted for (VAF) of the mean (VAFM) and variance (VAFV), which are defined as

$$
\begin{aligned}
\mathrm{VAFM} & =\left[1-\frac{(\overline{y(t)}-\overline{\hat{y}(t)})^{2}}{\overline{y(t)^{2}}}\right] \cdot 100 \% \\
\mathrm{VAFV} & =\left[1-\frac{\int_{0}^{L}(\tilde{y}(t)-\tilde{\hat{y}}(t))^{2} d t}{\int_{0}^{L} \tilde{y}^{2}(t)} d t\right] \cdot 100 \%
\end{aligned}
$$

where $\overline{y(t)}$ is the mean of $y(t)$ over time, $\tilde{y}(t)$ is $(y(t)-\overline{y(t)})$, and $\tilde{\hat{y}}(t)$ is $(\hat{y}(t)-\overline{\hat{y}}(t))$. VAFM and VAFV indicate how well the estimator predicts the mean and variation of the estimated signal. In the experiment only the VAFM and VAFV of the output can be calculated. Both VAFM and VAFV will be meaningless if their denominators are close to zero. For example, the inertia in the following simulation is assumed to be constant, $\tilde{y}(t)=0$. We suggest that when the denominator is less than one, we should set it to one. When a signal is zero mean, (9) is the same as the VAF definition in [14]. We require both VAFM and VAFV over 75\% [3], [14], for which the relative prediction error variance is less than one-third.

\section{B. Simulation Results}

In all of the simulations, the weighting factor $\lambda$ in (6) is $20 s^{-1}$. $\lambda$ determines the width of the weighting window in which the estimates are calculated as (6) indicates. We chose the window width five times smaller than the fast human arm movement, $4 \mathrm{~Hz}$ [2], [3]. A $10 \%-15 \%$ variation of $\lambda$ did not change the following simulated results much in the frequency range of interest.

The results for the FSEWLS tracking performance are summarized in Fig. 2 for posture and movement without derivative measurements. For the low-pass filter in (4), we use a third-order low-pass filter with a cutoff frequency of $3.2 \mathrm{~Hz}$, which is chosen based on the requirement of eight times reduction of the noise in the derivatives, assuming there is $5 \%$ noise over the signal standard deviation in the position measurement. For the high-pass filtering in (3), we use a fourth-order Chebyshev filter with a cutoff frequency of $1 \mathrm{~Hz}$. The lowest VAFM and VAFV are associated with the stiffness $K$, and are shown in Fig. 2 as the solid squares and solid circles. In all of the cases, the error is negative, i.e., the estimated parameter is smaller than the actual one. If the acceptable values for VAFM and VAFV are 80\%, from Fig. 2, we have the following observations.

- The FSEWLS tracking performance is better for posture $(1.5 \mathrm{~Hz})$ than for movement $(0.5)$ because the latter employs a high-pass filter (see Appendix B).

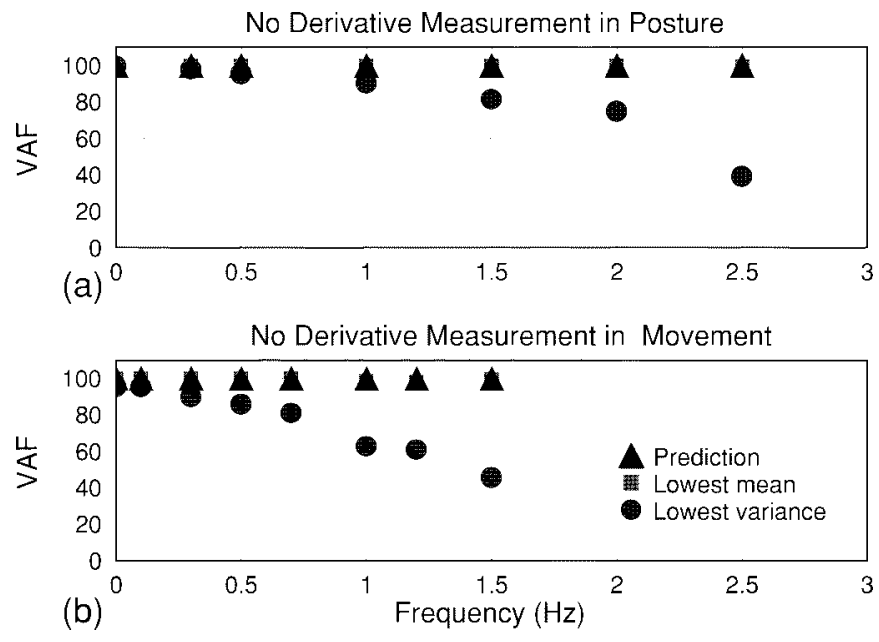

Fig. 2. Simulation: VAF of the output prediction, and the VAFM and VAFV of the stiffness $K$ versus the frequency of the parameter variation for posture and movement without derivative measurement.

- The VAF of the output prediction error is always high, close to $100 \%$ for all of the frequencies tested. This implies that the output prediction error is only a necessary condition for evaluating the parameter tracking performance.

Under our simulation conditions, the maximum parameter variation should not be more than $0.5 \mathrm{~Hz}$ as indicated in Fig. 2(b). The ratio of the parameter variation frequency to the filter cutoff frequency in (4) is about 6.4. Raising the filter cutoff frequency will improve the parameter tracking frequency, provided that the perturbation frequency and power are also increased to keep the noise-to-signal ratio (NSR = variance of noise/variance of signal) low.

The robustness of the FSEWLS method in the presence of output measurement noise is examined by simulating experiments with a variety of NSR without derivative measurements and in the presence of voluntary movement. Fig. 3 shows the variation of the VAF versus NSR. In general, the VAF of the output prediction and the parameter estimate decreases with the NSR. Note that the VAF of the parameter $K$ decreases faster than that of the output prediction, which indicates that the output prediction error is not a sufficient condition for measuring the method performance to noise. When NSR $=0.2$, the VAFV drops close to $80 \%$ for the simulated conditions.

\section{CALibration EXPERIMENTS}

The simulation study in the previous section is intended to find the conditions associated with the method under which the experiments must be controlled. The key conditions in our methods are: 1) the parameter variation must be less than $0.5 \mathrm{~Hz}$; 2) NSR must be less than 20\%; and 3) the perturbation model structure must be linear. Since the nature of the time-varying behavior of the neuromuscular system is still unknown, it is impossible to simulate it with great certainty. However, the magnitude of the changes and the time over which they occurred may be controlled to satisfy the conditions above. Consequently, we should be able to 

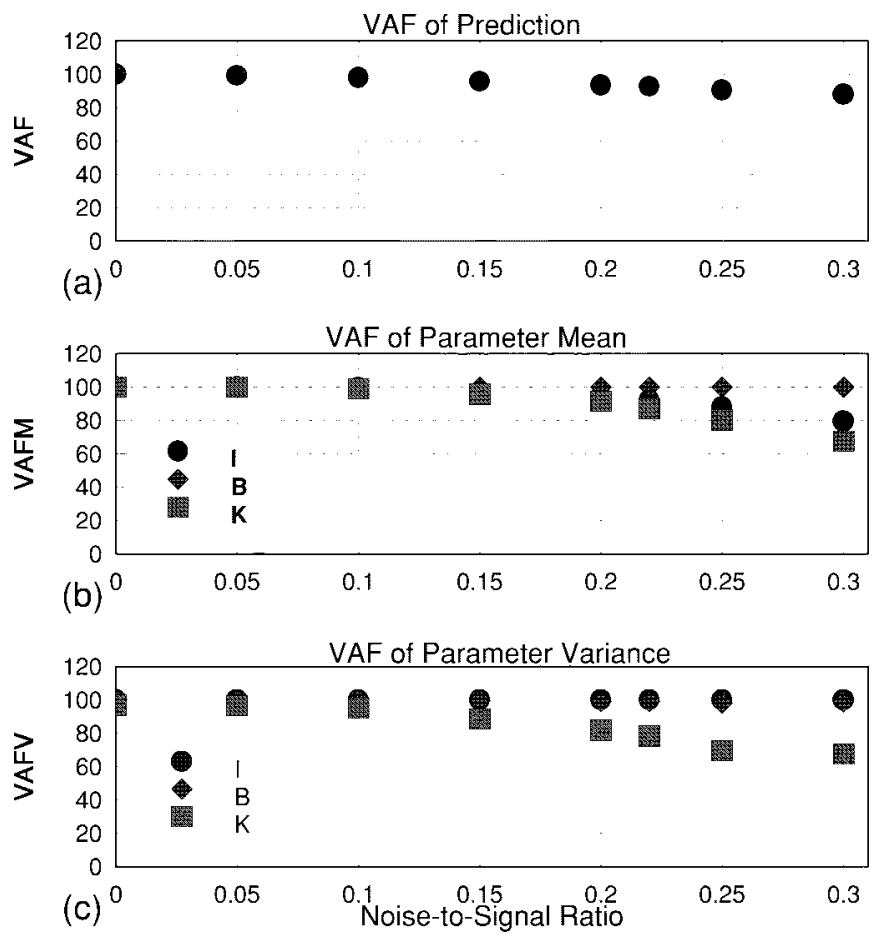

Fig. 3. Simulation: (a) VAF of the output prediction, (b) VAFM, and (c) VAFV of parameters versus the NSR of the output measurement noise.

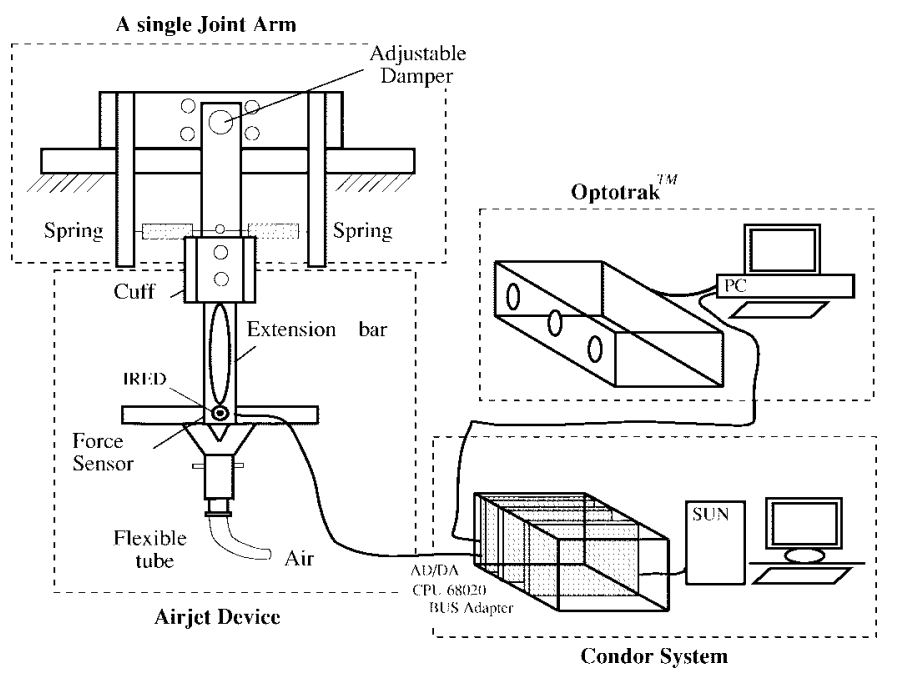

Fig. 4. Apparatus: an airjet system and a single joint, passive arm used for calibration.

obtain an approximate model with reasonable confidence. To demonstrate it, we did calibration experiments.

\section{A. Apparatus}

The calibration setup is shown in Fig. 4. We built a well calibrated single joint, mechanical arm which consists of an arm, two linear springs, and a joint made of a damper (KINETROL KD-2a). The damper has a shaft-mounted vane rotating between fixed vanes in the body. The motion is restricted to $\pm 45^{\circ}$. The springs are high precision with constant stiffness.

The airjet system [21] is a complete dynamical measuring system, capable of mechanical perturbation generation, motion tracking, and data acquisition. It consists of three parts: an airjet perturbation device, a motion tracking device, and a data acquisition system. The airjet device [20] has an airjet actuator, an extension bar, and a cuff (Fig. 4). The airjet actuator is currently a 1-D perturbation device, capable of producing $\pm 4 \mathrm{~N}$ forces from the reaction force of compressed air. The direction of the airflow could be switched $180^{\circ}$ in less than $5 \mathrm{~ms}$ by using a Coanda valve. The extension bar allows the airjet to be mounted distally to the hand so that the force is in the direction of elbow flexion/extension and is aligned with the long axis of the arm to avoid pronation/supination torques. The extension bar is supported by an aluminum cuff. The left wrist of each subject is custom fitted with a molded plastic cuff to which the aluminum cuff can be tightly clamped. The aluminum cuff can be tightened until movement from skin compliance is negligible for the perturbation used (NSR $<1 \%$ ). A strain gauge force sensor is mounted between the airjet actuator and the extension bar. The sensor has a resonance at $160 \mathrm{~Hz}$.

The motion tracking device, the Optotrak (Northern Digital Inc., Waterloo, Ont., Canada), uses three LCD cameras to track infrared light-emitting diodes (IRED's) and produces threedimensional position data of the IRED's with an accuracy of $0.05 \mathrm{~mm}$. All of our experiments were in a vertical plane, which is aligned with the Optotrak's $x-y$ internal plane. We used one IRED in the experiments, with a sampling rate of $500 \mathrm{~Hz}$. The Optotrak data is later resampled digitally to 1000 $\mathrm{Hz}$ to match the airjet force sampling rate.

The PRBS signal was generated digitally in real-time and fed to the airjet through a 12-bit digital-to-analog converter (DAC) at $100 \mathrm{~Hz}$. The airjet force signal was first amplified by a signal conditioner 2B31J from Analog Devices, then low-pass filtered by a linear phase analog filter DOW848 from Frequency Devices with a cutoff frequency of $150 \mathrm{~Hz}$ before digitization by a 12-bit analog-to-digital converter (ADC). The pure time delay introduced by the low-pass filter was corrected later, digitally. The sampling frequency was $1000 \mathrm{~Hz}$. The synchronization between the Optotrak and the real-time data acquisition system was made at the beginning of the data collection through an external triggering mechanism provided by the Optotrak.

\section{B. Calibration Results}

The model of the artificial arm is given by (7) with $\tau_{p}=0$. The parameter values in the model are calculated based on the geometry, material, and gravity properties: $I=0.0687 \mathrm{Nms}^{2} / \mathrm{rad}, B_{\min }=1.13 \mathrm{Nms} / \mathrm{rad}$, $B_{\max }=10.1 \mathrm{Nms} / \mathrm{rad}$, and $K=15.4 \mathrm{Nm} / \mathrm{rad}$.

The airjet device is mounted at the tip of the arm similar to the human wrist mounting. Fig. 5 shows the time-varying results, where the damping was varied during the experimental trial. Note that the inertia $I$ and stiffness $K$ do not vary and have estimation errors less than 5\%, and the output VAF is also $99 \%$. The results show that the apparatus as well as the method work as expected.

\section{Human SubJeCt EXPERIMENTS}

\section{A. Subjects}

Five healthy right-handed subjects (four males, one female), ranging in age from 22 to 26 years, were examined. Because 

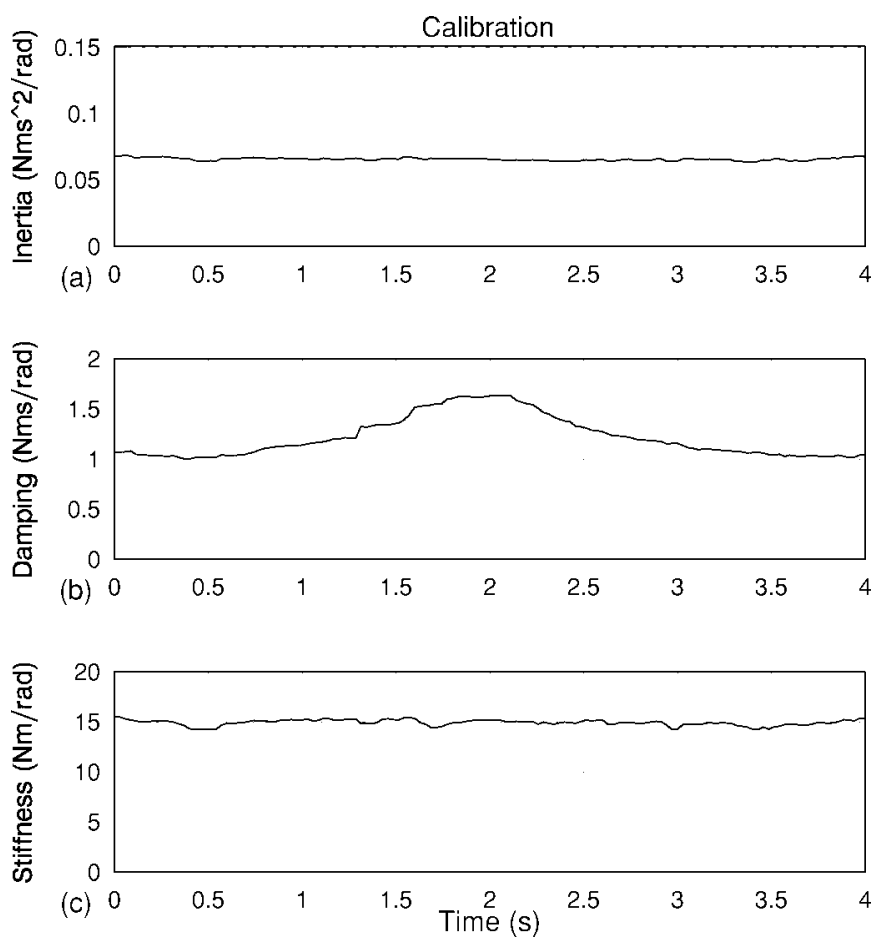

Fig. 5. Calibration: linear time-varying experiment. The damping was varied during the experiment.

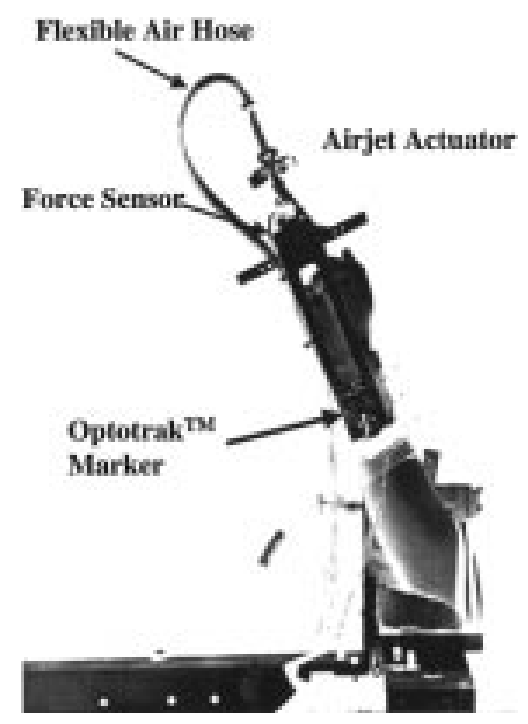

Fig. 6. The airjet actuator attached to a subject's wrist.

the results are similar across subjects, we only show the results from two subjects. However, the experimental conclusions are drawn based on the observation from all five subjects. Experiments were done with a subject's left arm. The subject sat in a chair and rested his elbow on a piece of clay on the bench. The airjet actuator was attached to the subject's wrist (Fig. 6). The elbow length was estimated by using both caliber measurement and the Optotrak. The results differ by less than $2 \%$. The forearm was free to move in a vertical plane passing through the upper arm. The subject's palm was turned $90^{\circ}$ away from his shoulder. The wrist was immobilized through a custom fitted plastic cuff.

\section{B. Posture Experiments}

The postural experiments were intended to examine the change of the mechanical properties for the elbow joint for different efforts of maintaining the posture and to test the FSEWLS method against existing methods. A different effort is an indication of a different co-contraction, and was realized by instructing a subject to use minimum, medium and maximum effort in maintaining his/her forearm posture against the perturbations. Furthermore, we tested the method's continuous tracking capability by asking subjects to vary the effort during a trial.

In all of our experiments, the torque perturbation is less than $5 \%$ of the normal elbow torque range $(26 \mathrm{Nm})$ and the movement is about $5 \%$ of the elbow joint movement range $\left(100^{\circ}\right)$. The small force perturbation is a prerequisite toward the linear identification of a nonlinear biological system.

1) Time-Invariant Posture and Linearity Test: Because the results are consistent with previous findings, we merely state them here. For all of the experiments, the VAF of the output prediction is over $95 \%$

- The inertia for each subject is approximately constant (less than $1 \%$ change) over time and does not vary with different effort. Across subjects, it may vary by $80 \%$.

- The damping drifts slowly over time and increases with effort for all subjects. Across subjects, it may vary by $100 \%$.

- The stiffness drifts over time and increases with the effort, similar to that of the damping. Across subjects it can vary by ten times.

- The damping ratio drifts as well and does not vary much across effort.

In order to test the linearity of the elbow joint under small perturbation, on one of the subjects (RSG) a set of the experiments was conducted with lower air pressure (45 psi), so that the force perturbation amplitude was reduced to half $(2 N)$. There is little difference in terms of the profiles for all of the parameters. The difference of the mean value of the parameters is within $5 \%$, which may well be due to intertrial variation. The VAF's for both cases are above 95\%. This indicates the validity of the linearization under the experimental conditions.

2) Time-Varying Posture: A moving target was presented to the subject during each trial. When the target was moving forward, the subject gradually increased the effort, and vice versa. Fig. 7 shows the measurements of the elbow joint angles and perturbation torques. The maximum joint angle is about $0.058 \mathrm{rad}\left(3.32^{\circ}\right)$, and the maximum perturbation torque to the elbow joint is about $2.4 \mathrm{Nm}$. Fig. 8 shows the results of time-varying posture. The VAF for all of the experiments is over 95\%. From Fig. 8, we have the following observations.

- The inertia for each subject is approximately constant with time, and the differences across subjects are similar to those in the time-invariant posture experiments.

- The stiffness and damping vary dramatically with time and have the same variation pattern for all of the subjects. The values of the peaks and valleys are comparable to those from the time-invariant postural experiments with the minimum and maximum efforts. 

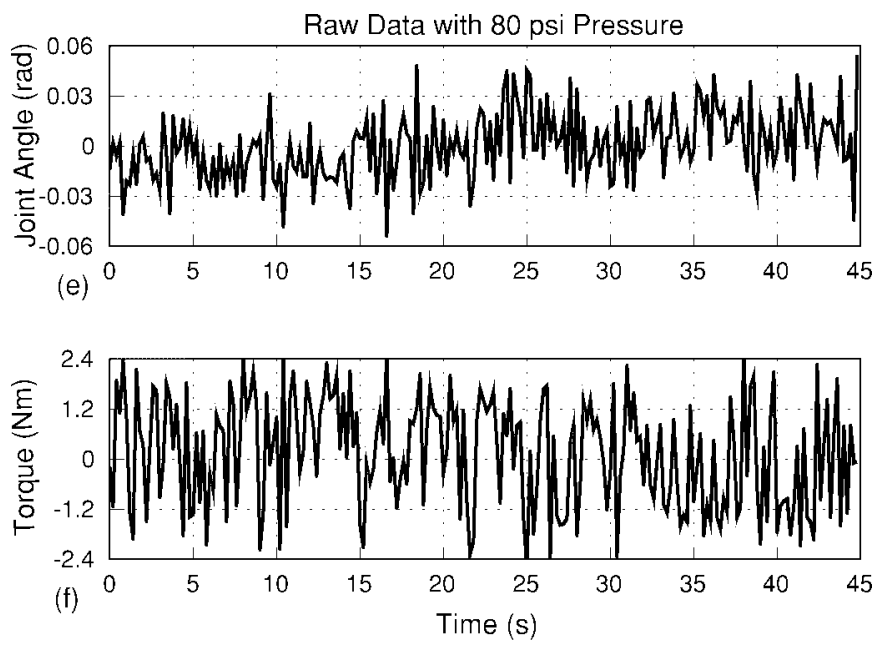

Fig. 7. Joint movement and perturbation torque measurements from postural experiment (subject $\mathrm{PAB}$ ).
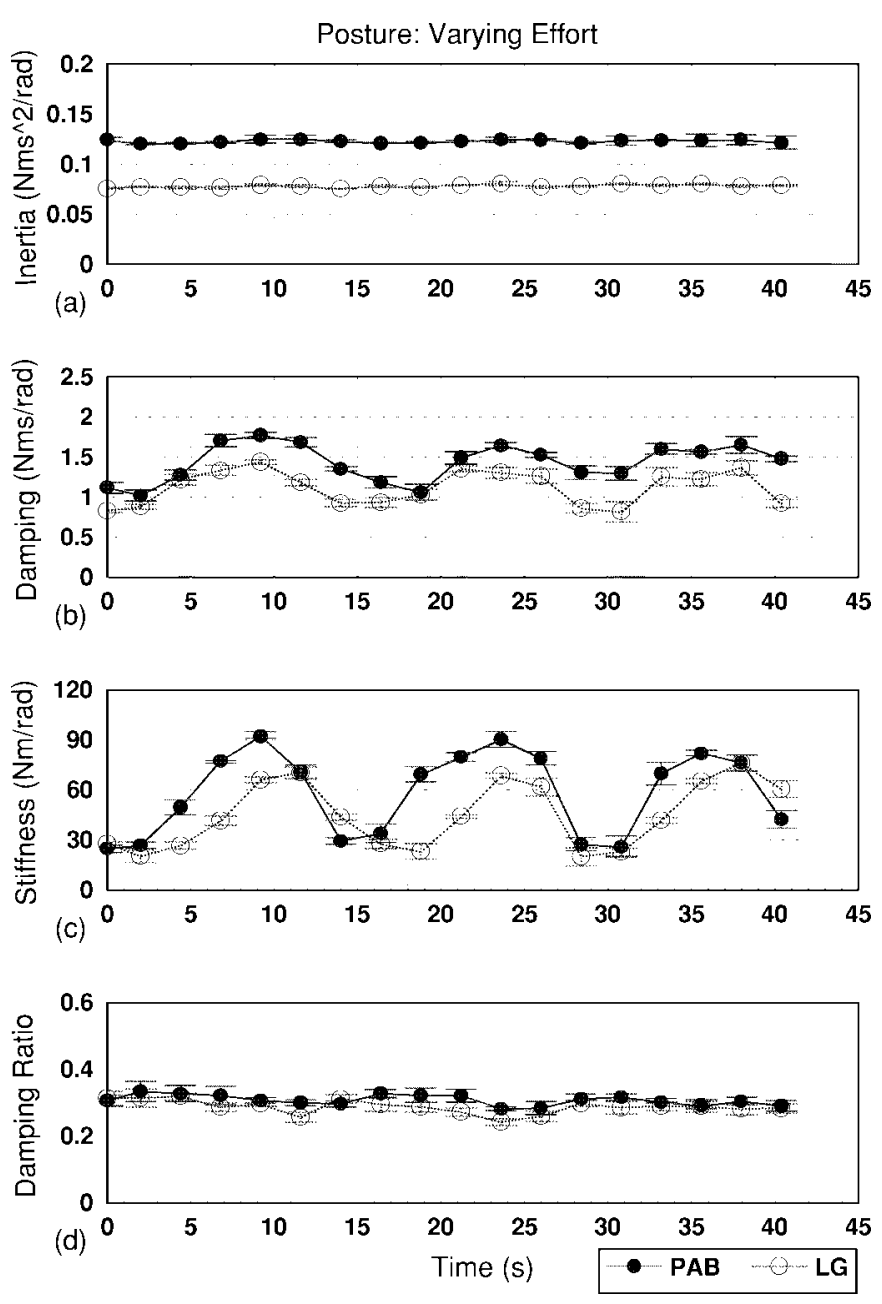

Fig. 8. Results from postural experiment (subjects PAB and LG): (a) inertia, (b) damping, (c) stiffness, and (d) damping ratio parameters with time-varying effort.

- The damping ratio for every subject drifts with time, but does not have a clear pattern. The mean value is between 0.1 and 0.5 , and similar to that in the time-invariant postural experiments.
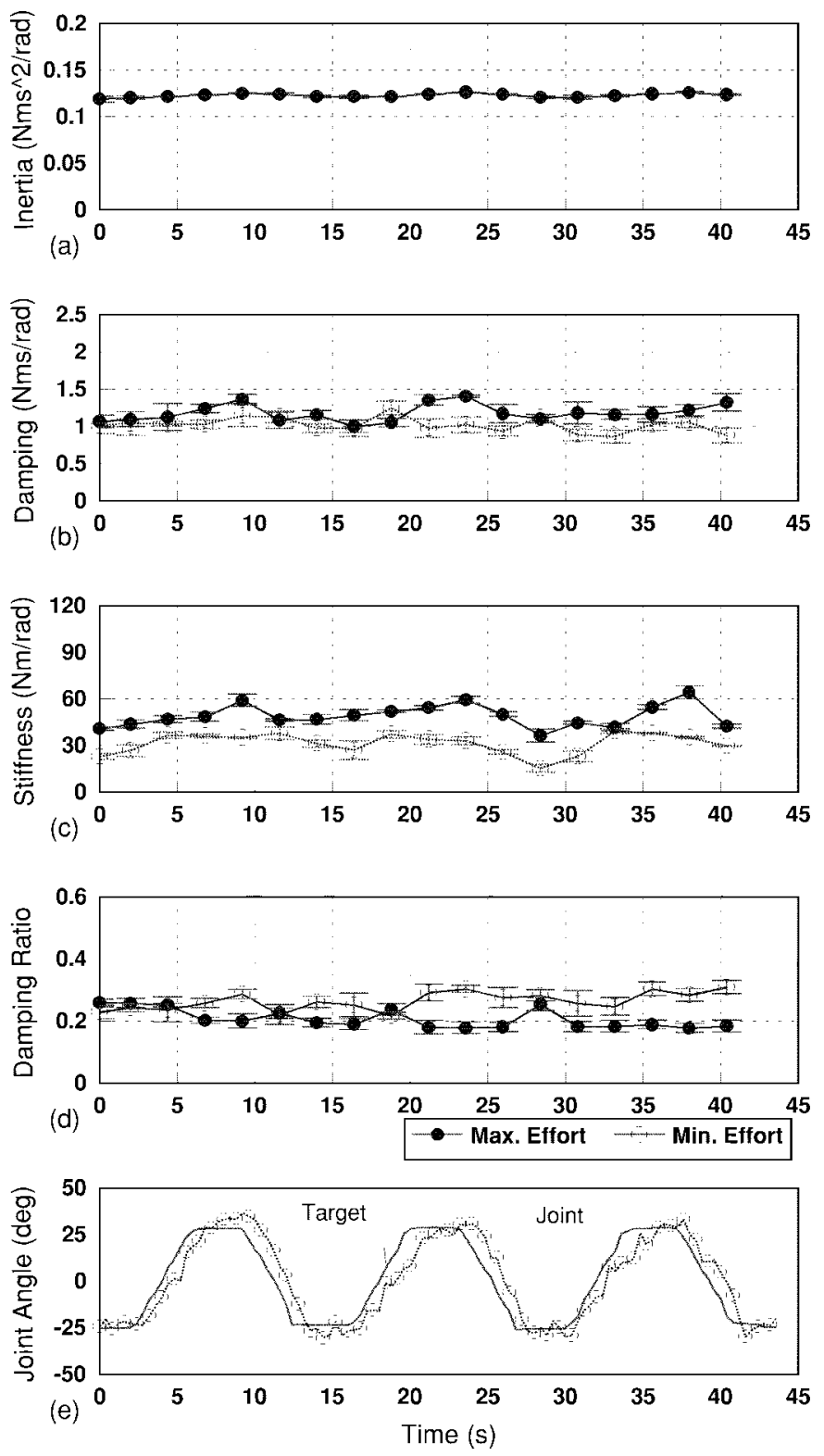

Fig. 9. Results from movement experiments with minimum and maximum efforts from subject PAB: (a) inertial, (b) damping, (c) stiffness, (d) damping ratio parameters in minimum effort tracking task, and (e) shows the actual movement trajectories.

\section{Movement Experiments}

The voluntary movement in the experiments is slow so that the mechanical properties also change slowly. In the experiments, we presented a slow-moving target to the subject. The subject was instructed to track the target with a reasonable accuracy. The result from subject PAB is shown in Fig. 9. The inertia for minimum and maximum efforts differs by less than $2 \%$. The target trajectory is given in Fig. 9(e). Each movement phase (4s) is followed by a postural phase ( $3 \mathrm{~s})$. In each trial, there are seven posture and six movement phases in $45 \mathrm{~s}$. The maximum movement speed is $15 \%$, and the movement frequency is about $0.071 \mathrm{~Hz}$. If we do not consider the postural phases, the frequency of $0.125 \mathrm{~Hz}$ is well below the $0.5 \mathrm{-Hz}$ boundary frequency of the FSEWLS method. The VAF for all of the trials is above $95 \%$. 


\section{1) Inertia:}

- The inertia is approximately constant with a variation of less than $5 \%$.

\section{2) Damping:}

- At a constant effort, the damping drifts without a clear correlation to the movement. The mean and variation magnitude are in the same range as in the time-invariant postural experiments.

- The damping variation with effort is similar to that in the time-invariant posture experiments. At the maximum effort, the variation is slightly smaller than that in posture.

- The damping with the maximum effort is higher than that with the minimum effort. The mean value is about 0.1 $\mathrm{Nms} / \mathrm{rad}$ higher. However, in the varying effort posture experiment (Fig. 8), the damping varies by around 1.7.

- Across subjects, the variation is also similar to that in the time-invariant posture experiments.

3) Stiffness:

- The stiffness variation is similar to the damping variation. The stiffness variation is not strongly correlated with the movement. The stiffness is higher with the maximum effort than that with the minimum effort. The mean value differs by about $10 \mathrm{Nm} / \mathrm{rad}$.

4) Damping Ratio:

- The damping ratio variation is similar to the damping variation as well. The mean value with the minimum effort is a little higher than that with the maximum effort, opposite to the damping. This indicates the damping plays more of a role in the minimum effort case.

\section{DISCUSSION}

This paper demonstrates that the FSEWLS method is a successful means to identify the slow time-varying dynamics of the human motor control system. The key in the method is to eliminate the voluntary response from raw data through high-pass filtering and to estimate the derivatives through low-pass filtering. The method has three major advantages: 1) it works on a single trial; 2) it has few restrictions on the input signal; and 3) it has a well-characterized error. Under our experimental conditions (small force perturbation, no derivative measurements, and unknown voluntary actions), the technique estimates the time-varying parameters up to a frequency of $0.5 \mathrm{~Hz}$. The method is quite robust to output noise and has been tested extensively. Because the FSEWLS method is capable of tracking dynamical change in posture, it may be used clinically to do monitoring and to evaluate functional electrical stimulation in rehabilitation engineering.

Recent advances in ensemble-based methods have reduced their sensitivity to intertrial variation [19]. However, they must have fast movement patterns in order to align trials. We think that the FSEWLS and robust ensemble methods can complement each other, in that the FSEWLS method works in posture and slow movement domains while ensemble methods work in fast movement domains.
One of the major goals in this study has been to investigate the limitations of the FSEWLS method and to establish the applicable boundaries under the experimental conditions. We discovered that the output VAF cannot be used exclusively to judge how good the parameter estimates are. Theoretical analysis, simulation, and experimental calibration are necessary to find the application boundaries for accurate estimates.

\section{A. Physiological Results}

The primary objective of the experimental study was to establish the viability of the FSEWLS method. Nevertheless, the results are of considerable physiological interest. The time course of the dynamic change of the elbow joint in posture has not been reported previously. Previous results are the average values over a certain period of time.

We discovered that at a fixed effort the stiffness, damping and damping ratio can vary with time while the inertia is constant. The variation of the damping ratio is smaller than that of the stiffness or damping, but is significant. In timevarying posture, the damping variation with stiffness has been demonstrated. The damping ratio shows no clear correlation with stiffness or damping even though it is computed from them. This brings up the question of whether the stiffness and damping are controlled independently or the damping ratio is the controlled quantity. In any case, the damping ratio is between 0.1 and 0.5 , which indicates that the joint is underdamped.

The results from movement are different from previous ones with the normal or fast movements. The stiffness, damping and damping ratio are all in the same range as those from the postural experiments, much higher than reported before [3]. Furthermore, their variations over time do not show a strong correlation with the movement. One reason may be due to the slow movements in the current experiments. If both results are accurate, it may indicate that there is a significant decrease of the stiffness and damping from very slow to normal or fast movements. On the other hand, the current movement experiments show that there is no sudden change of mechanical properties from posture to movement. Flash [5] showed, in simulation, that in order to fit the hand trajectory well during normal movements, the joint stiffnesses must be equal to or higher than those in posture. Further experiments are needed to resolve this difference.

\section{B. Comparison with Previous Studies}

There are several published articles on time-varying dynamics of the human forearm system, which are all ensemble-based methods. There are no reports on their sensitivity to intertrial variations. Soechting et al. [17] devised a correlation-based method to study the changes in human myotactic reflex (dynamic relationship between joint angular position and surface electromyographic activity). Lacquaniti et al. [10] applied the same method to studying the human elbow compliance during a change in contraction level of the muscles of the upper arm. The impulse functions were fitted by a secondorder model to obtain compliance parameters. The inertia parameter was not estimated based on their experimental data, 
but on anthropometric tables. Our result shows that the inertia varies significantly across subjects $(100 \%)$. Nevertheless time-invariant results from our methods agree with theirs qualitatively.

Bennett et al. [3] devised an ensemble parametric method to identify the time-varying compliance of the human elbow joint during normal voluntary movements; the cyclic voluntary movement frequency was about $1 \mathrm{~Hz}$, ten times faster than the movement used here. A linear second-order model was used to fit the data. The method does not use correlation functions and, therefore, does not have many requirements on the input perturbation as does Soechting's. The mean value of stiffness was very low ( $\leq 8 \mathrm{Nm} / \mathrm{rad})$, much lower than that from posture with minimum effort. The stiffness was found lower during movement than that at target points. More recently Bennett [2] showed with pulse perturbation that the stiffness increases with movement speed. The stiffness level is also below those in posture.

MacNeil et al. [14] devised an ensemble nonparametric method to study the time-varying dynamics during rapid voluntary force change in the human ankle joint. They found that during the force ramping, the ankle joint cannot be modeled by a second-order system and the dynamic stiffness decreased with the mean force, which contradicts the postural results in [7].

\section{Nonlinear Considerations}

The biological system is inherently nonlinear. If the system is assumed to be nonlinear in general and the voluntary action is not measured completely, the perturbation approach has to be devised carefully so that the linearization is correctly realized, since superposition is explicitly or implicitly used in all of these approaches. For example, in the movement experiments, we must subtract the mean from the raw data in order to obtain the perturbed responses. The part of the mean (especially the time-varying mean) is due to voluntary actions. On the other hand, by understanding the linearized dynamics of a nonlinear system, we can gain considerable knowledge about the system. Better experiments may be devised based on this knowledge. This is the exact drive behind this study. Indeed, the results of this study may be considered to be our first step toward the identification of the underlying nonlinear biological system.

\section{APPENDIX A}

EFFECT OF LOW-PASS FILTERING ON PARAMETER ESTIMATION

Consider a general time-varying input and output system

$$
\begin{aligned}
& a_{n}(t) \theta^{(n)}(t)+\cdots+a_{1}(t) \dot{\theta}(t)+a_{0}(t) \theta(t) \\
& \quad=b_{n-1}(t) \tau^{(n-1)}(t)+\cdots+b_{1} \dot{\tau}(t)+b_{0}(t) \tau(t)
\end{aligned}
$$

where $\tau$ and $\theta$ are the input and output variables, and the $a$ 's and $b$ 's are time-varying coefficients. Apply a linear filter (4)

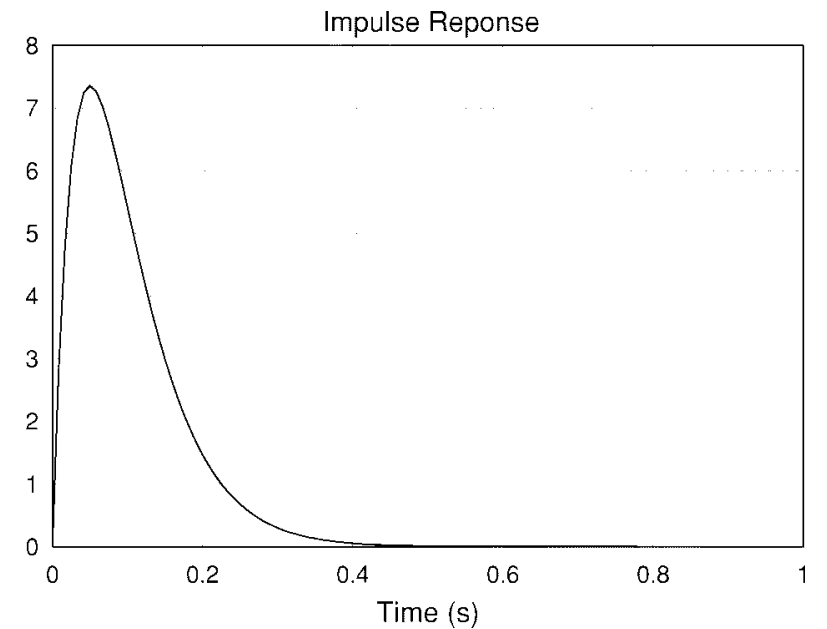

Fig. 10. Simulation: impulse response function from a recursive low-pass filter.

to the input and output signals

$$
\begin{aligned}
\theta_{L}(t)= & \int_{0}^{t} l(t-\epsilon) \theta(\epsilon) d \epsilon \\
\dot{\theta}_{L}(t)= & \int_{0}^{t} l(t-\epsilon) \dot{\theta}(\epsilon) d \epsilon+l(t) \theta(0) \\
& \vdots \\
\theta_{L}^{(n)}(t)= & \int_{0}^{t} l(t-\epsilon) \theta^{n}(\epsilon) d \epsilon+l^{n-1}(t) \theta(0)+\cdots+l(t) \theta(0) \\
\tau_{L}(t)= & \int_{0}^{t} l(t-\epsilon) \tau(\epsilon) d \epsilon \\
\dot{\tau}_{L}(t)= & \int_{0}^{t} l(t-\epsilon) \dot{\tau}(\epsilon) d \epsilon+l(t) \tau(0) \\
& \vdots \\
\tau_{L}^{(n)}(t)= & \int_{0}^{t} l(t-\epsilon) \tau^{n}(\epsilon) d \epsilon+l^{n-1}(t) \tau(0)+\cdots+l(t) \tau(0)
\end{aligned}
$$

where $l(\cdot)$ is the impulse response of the low-pass filter $L(s)$, $L(0)=0$ and $L(\infty)=0$. A typical impulse response of a second-order low-pass filter with damping ratio at one is given in Fig. 10. Because the filter is an infinite impulse filter (or recursive filter) $l(0)=0$ and $l(t) \approx 0$ if $t>1 / \omega$. Therefore, the terms with $l(t)$ in the above expressions should be zero when $t$ is sufficiently large. If all of the coefficients $a$ 's and $b$ 's are constant then

$$
\begin{aligned}
& \int_{0}^{t} l(t-\epsilon)\left[a_{n} \theta^{(n)}(\epsilon)+\cdots+a_{1} \dot{\theta}(\epsilon)+a_{0} \theta(\epsilon)\right] d \epsilon \\
& \quad=\int_{0}^{t} l(t-\epsilon)\left[b_{n-1} \tau^{(n-1)}(\epsilon)+\cdots+b_{1} \dot{\tau}(\epsilon)+b_{0} \tau(\epsilon)\right] d \epsilon
\end{aligned}
$$

after $t$ is sufficiently large. This means that the identification method described in Section II-C is the exact solution. If the coefficients $a$ and $b$ are time-varying but approximately constant within the time constant of the low-pass filter, then (11) will still be valid. This means that the ratio of the frequencies of the parameter variation and the low-pass filter must be smaller than one. The filter bandwith does not have to be higher than the bandwith of the system as (11) suggests. 
However, the low filter bandwith will decrease the signal-tonoise ratio of the derivative terms. A simulation not shown here indicates that the reliable parameter estimates can be obtained even if the filter bandwidth is lower than that of the system with PRBS perturbation. When the bandwith of a system dynamics is known only approximately, it is safe to have the filter bandwith higher than that of the system dynamics.

\section{APPENDIX B}

\section{EFFECT OF HIGH-PASS FILTERING ON PARAMETER ESTIMATION}

Consider the linearized dynamics of the elbow joint in (2). Apply a high-pass filter (3) to the measurements $\theta_{\text {meas }}(t)$ and $\tau_{\text {meas }}(t)$

$$
\begin{aligned}
\delta \tilde{\theta}(t) & =\theta_{\text {meas }}(t)-\int_{-\infty}^{\infty} a l(t-\epsilon) \theta_{\text {meas }}(\epsilon) d \epsilon \\
\tilde{\tau}(t) & =\tau_{\text {meas }}(t)-\int_{-\infty}^{\infty} a l(t-\epsilon) \tau_{\text {meas }}(\epsilon) d \epsilon
\end{aligned}
$$

where $q(\cdot)$ is the impulse function of the acausal low-pass filter (4). The derivatives are

$$
\begin{aligned}
& \delta \dot{\tilde{\theta}}(t)=\dot{\theta}_{\text {meas }}(t)-\int_{-\infty}^{\infty} q(t-\epsilon) \dot{\theta}_{\text {meas }}(\epsilon) d \epsilon \\
& \delta \ddot{\tilde{\theta}}(t)=\ddot{\theta}_{\text {meas }}(t)-\int_{-\infty}^{\infty} q(t-\epsilon) \ddot{\theta}_{\text {meas }}(\epsilon) d \epsilon .
\end{aligned}
$$

Now consider the difference and assume that the voluntary movement is low-frequency only

$$
\begin{aligned}
& {[I(t) \delta \ddot{\tilde{\theta}}(t)+B(t) \dot{\tilde{\theta}}(t)+K(t) \delta \tilde{\theta}(t)]-\tilde{\tau}(t)} \\
& =[I(t) \ddot{\theta}(t)+B(t) \dot{\theta}(t)+K(t) \theta(t)-\tau(t)] \\
& +\left[\int_{-\infty}^{\infty} a l(t-\epsilon) \tau(\epsilon) d \epsilon-I(t) \int_{-\infty}^{\infty} a l(t-\epsilon) \ddot{\theta}(\epsilon) d \epsilon\right. \\
& -B(t) \int_{-\infty}^{\infty} a l(t-\epsilon) \dot{\theta}(\epsilon) d \epsilon \\
& \left.-K(T) \int_{-\infty}^{\infty} a l(t-\epsilon) \theta(\epsilon) d \epsilon\right] \\
& =I(t)\left[\ddot{\theta}_{n}(t)-\int_{-\infty}^{\infty} a l(t-\epsilon) \ddot{\theta}_{n}(\epsilon) d \epsilon\right] \\
& +B(t)\left[\dot{\theta}_{n}(t)-\int_{-\infty}^{\infty} a l(t-\epsilon) \dot{\theta}_{n}(\epsilon) d \epsilon\right] \\
& +K(t)\left[\theta_{n}(t)-\int_{-\infty}^{\infty} a_{l}(t-\epsilon) \theta_{n}(\epsilon) d \epsilon\right] \\
& +[I(t) \delta \ddot{\theta}(t)+B(t) \delta \dot{\theta}(t)+K(t) \delta \theta(t)-\tau(t)] \\
& +\left[\int_{-\infty}^{\infty} q_{l}(t-\epsilon) \tau(\epsilon) d \epsilon-\int_{-\infty}^{\infty} a_{l}(t-\epsilon) I(t) \delta \ddot{\theta}(\epsilon) d \epsilon\right. \\
& -\int_{-\infty}^{\infty} q_{l}(t-\epsilon) B(t) \delta \dot{\theta}(\epsilon) d \epsilon \\
& \left.-\int_{-\infty}^{\infty} a l(t-\epsilon) K(t) \delta \theta(\epsilon) d \epsilon\right] \\
& \approx \int_{-\infty}^{\infty}{ }^{l} l(t-\epsilon)[\tau(\epsilon)-I(t) \dot{\delta} \ddot{\theta}(\epsilon)-B(t) \delta \dot{\theta}(\epsilon) \\
& -K(t) \delta \theta(\epsilon)] d \epsilon
\end{aligned}
$$

which shows that the high-pass filtering does not introduce error in the parameter estimation if the high-pass filter cutoff frequency is higher than that of the voluntary movement and the perturbation has sufficient power in the high frequency range. Given a type and amplitude of a perturbation, the higher the high-pass filter cutoff frequency, the less the useful perturbation frequency range, the less the perturbation power, the higher the NSR and the estimation error. This is where the source of the error is with the high-pass filtering method.

\section{REFERENCES}

[1] K. J. Astrom and B. Wittenmark, Adaptive Control. New York: Addison-Wesley, 1988

[2] D. J. Bennett, "Torques generated at the human elbow joint in response to constant position errors imposed during voluntary movements," Exp. Brian Res., vol. 88, pp. 433-442, 1992.

[3] D. J. Bennett, J. M. Hollerbach, I. W. Hunter, and Y. Xu, "Time-varying stiffness of human elbow joint during cyclic voluntary movement," Exp. Brian Res., vol. 95, pp. 488-498, 1993.

[4] J. E. Colgate, "The design of a dynamics measuring device," Master's thesis, Mech. Eng. Dept., Massachusetts Inst. Technol., Cambridge, 1986

[5] T. Flash, "The control of hand equilibrium trajectories in multi-joint arm movement," Biological Cybern., vol. 57, pp. 257-274, 1987.

[6] C. G. Goodwin and K. S. Sin, Adaptive Filtering, Prediction and Control. Englewood Cliffs, NJ: Prentice-Hall, 1984.

[7] I. W. Hunter and R. E. Kearney, "Dynamics of human ankle stiffness Variation with mean ankle torque," J. Biomech., vol. 15, pp. 747-752, 1982.

[8] R. E. Kearney and I. W. Hunter, "Dynamics of human ankle stiffness: Variation with displacement amplitude," J. Biomech., vol. 15, pp 753-759, 1982.

[9] _ "System identification of human joint dynamics," Crit. Rev. Biomed. Eng., vol. 18, no. 1, pp. 55-87, 1990

[10] F. Lacquaniti, F. Licata, and J. F. Soechting, "The mechanical behavior of the human forearm in response to transient perturbations," Biological Cybern., vol. 44, pp. 35-46, 1982.

[11] J. M. Lanman, "Movement and the mechanical properties of the intact human elbow joint," Ph.D. dissertation, Psych. Dept., Massachussets Int. Technol., Cambridge, 1980.

[12] L. Ljung, System Identification: Theory for the User, Englewood Cliffs, NJ: Prentice-Hall, 1987.

[13] L. Ljung and T. Söderström, Theory and Practice of Recursive Identification. Cambridge, MA: MIT Press, 1983.

[14] J. B. MacNeil, R. E. Kearney, and I. W. Hunter, "Identification of timevarying biological systems from ensemble data," IEEE Trans. Biomed. Eng., vol. 39, pp. 1213-1225, Dec. 1992.

[15] R. B. Stein, R. E. Kearney, and L. Parameswaran, "Identification of intrinsic and reflex contributions to human ankle stiffness dynamics," IEEE Trans. Biomed. Eng., vol. 44, pp. 493-504, June 1997.

[16] J. J. Slotine and W. Li, Applied Nonlinear Control. Englewood Cliffs, NJ: Prentice Hall, 1991

[17] J. F. Soechting, J. R. Dufresne, and F. Lacquaniti, "Time-varying properties of the myostatic response in man during some simple motor tasks," J. Neurophysiol., vol. 46, no. 6, pp. 1226-1243, 1981.

[18] Y. Xu and J. M. Hollerbach, "Design and calibration of a 2-D airjet device for human motor control study," presented at 17th Annu. Conf. IEEE Eng. in Medicine and Biol. Soc., Baltimore, MD, 1995.

[19] _ "Nonlinear time-varying identification of human arm joint mechanical properties using a portable pneumatic thruster," in Proc. 1997 American Control Conf., June 1997, pp. 3281-3285.

[20] Y. Xu, J. M. Hollerbach, and I. W. Hunter, "Time-varying identification of elbow joint dynamics using exponentially weighted least squares," in Proc. 13th Annu. Conf. IEEE Eng. in Med. and Biol. Soc., Orlando, FL, Nov. 1991, pp. 2020-2021.

[21] Y. Xu, I. W. Hunter, J. M. Hollerbach, and D. J. Bennett, "An airjet actuator system for identification of the human arm joint mechanical properties," IEEE Trans. Biomed. Eng., vol. 38, pp. 1111-1122, Nov. 1991.

[22] V. A. Yosef and G. F. Inbar, "Parameter estimation of the mechanical impedance of the forearm of the human operator using Gaussian torque input," Technion-Israel Institute of Technology, Dept Elec. Eng., Haifa, Israel, 1988, Tech. Rep. 582. 


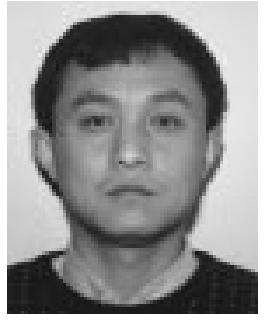

Yangming Xu (S'90-M'91) received the B.S. degree in mechanical engineering from Northwestern Polytechnical University, China, in 1982, and the M.S. and Ph.D. degrees in aeronautics and astronautics from Massachusetts Institute of Technology, Cambridge, in 1986 and 1991, respectively.

$\mathrm{He}$ joined Sarcos Research Corporation (SRC), Salt Lake City, UT, in 1994 as a Senior Control/Software Engineer working in the fields of real-time control of robotic systems, whole-body virtual interface, tactile display, and high-speed sensor network. He is also a Research Assistant Professor at the Department of Mechanical Engineering, University of Utah, Salt Lake City. Before jointing SRC, he was a Postdoctoral Fellow in the Biomedical Laboratory, McGill University, Montreal, PQ, Canada. His research interests include whole body interface to virtual world, human motor control, system identification, control of biological/robotic systems, and intelligent sensor network.

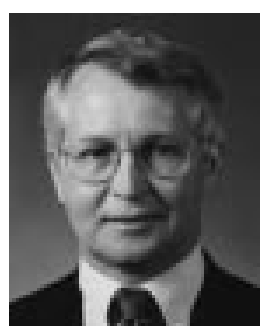

John M. Hollerbach (M'85-SM'92-F'96) received the B.S. degree in chemistry in 1968 and the M.S. degree in mathematics in 1969 , both from the University of Michigan, Ann Arbor. He received the S.M. degree in 1975 and the Ph.D. degree in 1978 , in computer science, both from Massachussets Institute of Technology (MIT), Cambridge.

$\mathrm{He}$ is Professor of Computer Science and Research Professor of Bioengineering and of Mechanical Engineering, at the University of Utah. From 1989-1994, he was with the Natural Sciences and Engineering/Canadian Institute for Advanced Research Professor of Robotics at McGill University, jointly in the Departments of Mechanical Engineering and Biomedical Engineering. From 1982-1989, he was on the faculty of the Department of Brain and Cognitive Sciences and a member of the Artificial Intelligence Laboratory at MIT; from 1978-1982, he was a Research Scientist. His research interests combine robotics, human motor control, teleoperation, virtual reality, and microelectromechanical systems. Presently, he is a Senior Editor of Presence: Teleoperators and Virtual Environments (Cambridge, MA: MIT Press), an Editorial Board Member of the International Journal of Robotics Research (Thousand Oaks, CA: Sage), and a Governing Board Member of the electronic journal, Haptics-e (http://www.haptics-e.org).

In 1984, Dr. Hollerbach received an NSF Presidential Young Investigator Award and in 1988, he was named a fellow of the Canadian Institute for Advanced Research. He was the Program Chairman of the 1989 IEEE International Conference on Robotics and Automation, a member of the Administrative Committee of the IEEE Robotics and Automation Society from 1989-1993. He served as Technical Editor of the IEEE TRANSACTIONS ON ROBOTICS AND AUTOMATION from 1989-1994 and Treasurer of the IEEE/ASME Journal of Microelectromechanical Systems, from 1992-1997. He was a Member of the 1994-1995 National Research Council Committee on Virtual Reality Research and Development. 